NBI-HE-00-10

\title{
Macroscopic and Microscopic (Non-)Universality of Compact Support Random Matrix Theory
}

\author{
G. $\operatorname{AKEMAnN}^{(1)}$ AND G. VERnizZI ${ }^{(2)}$ \\ (1) Max-Planck-Institut für Kernphysik \\ Saupfercheckweg 1, D-69117 Heidelberg, Germany \\ and \\ (2) The Niels Bohr Institute \\ Blegdamsvej 17, DK-2100 Copenhagen Ø, Denmark
}

\begin{abstract}
A random matrix model with a $\sigma$-model like constraint, the restricted trace ensemble (RTE), is solved in the large- $n$ limit. In the macroscopic limit the smooth connected two-point resolvent $G(z, w)$ is found to be nonuniversal, extending previous results from monomial to arbitrary polynomial potentials. Using loop equation techniques we give a closed though nonuniversal expression for $G(z, w)$, which extends recursively to all higher $k$ point resolvents. These findings are in contrast to the usual unconstrained one-matrix model. However, in the microscopic large- $n$ limit, which probes only correlations at distance of the mean level spacing, we are able to show that the constraint does not modify the universal sine-law. In the case of monomial potentials $V(M)=M^{2 p}$, we provide a relation valid for finite- $n$ between the $k$-point correlation function of the RTE and the unconstrained model. In the microscopic large- $n$ limit they coincide which proves the microscopic universality of RTEs.
\end{abstract}




\section{Introduction}

Random matrix models enjoy a wide range of applications in physics due to their property of being universal (for reviews see [1, 2]). This property manifests itself in the independence of correlation functions from the choice of the distribution function $\mathcal{P}(M) \sim \exp [-n \operatorname{Tr} V(M)]$, where $M$ is an $n \times n$ matrix and $V$ is a polynomial. Different classes of universality are found

depending on the way the large- $n$ limit is taken [3, 4, 5] and which part of the spectrum is investigated [5, 6, 0, 8]. However, not in all applications a distribution function of the above form is realistic. Here, all the eigenvalues (=energy levels) of the matrix $M$ are coupled through the Jacobian after diagonalization. There are situations as for example in applications in Nuclear Physics, where in contrast to that the Hamiltonian of the model couples only few energy levels and is still very well described by the above random matrix model [1]. It is therefore very interesting to study deformations and generalizations of the above distribution $\mathcal{P}(M)$ and to investigate first, if the correlations remain unchanged and second, if the property of universality is maintained. In this work we study a deformation which preserves the symmetry of the matrix model, which will be the unitary transformations of the Hermitian matrix $M$ in our case. The symmetry of the model is directly related to the properties of the Hamiltonian under rotations and time-reversal [1]. There exists an interesting relation [9] between the restricted trace ensembles (RTEs) which we will consider and the so-called Wigner ensembles [10, 11], where different matrix elements are weighted with different distribution functions, without being invariant under unitary transformations.

It has been known only quite recently that there exist symmetry preserving deformations of the distribution function that destroy the property of universality [9, 12]. Two examples are the trace squared ensembles which were originally introduced in the context of Quantum Gravity [13, 14] and the generalized RTEs which were introduced, in their simplest purequadratic form, by Bronk and Rosenzweig [15, 16] in the context of Nuclear Physics. The non-universality does not necessarily spoil the applicability to physical systems. In fact the deformation may be introduced for physical reasons: indeed, trace-squared terms have been recognized as corresponding to higher order intrinsic curvature terms in the string action. Such terms have been added in order to cure an intrinsic instability of the theory related to a crumpled surface phase of the string world sheet [13, 14, 17]. Moreover, in the framework of random surfaces, these additional terms are interpreted 
as touching interaction terms which make the random surfaces touch each other. The generalized RTEs permit the same graphical interpretation as they have been shown to be a limiting case of a special trace squared ensemble [18]. The RTEs are defined such that the exponential weight function gets replaced by a constraint $\mathcal{P}_{\delta}(M) \sim \delta\left(A^{2}-1 / n \operatorname{Tr} V(M)\right.$ ) (or the $\theta$ Heaviside step function instead). By using the following representation of the $\delta$-function, $\delta(x)=\lim _{l \rightarrow \infty} \sqrt{\pi / l} \exp \left[-l x^{2}\right]$, the distribution $\mathcal{P}_{\delta}(M)$ can be easily seen to contain trace squared terms. The relation between RTEs and the trace squared ensembles was discussed in great detail for the spectral density in [18] using saddle point techniques, and the canonical ensemble, the trace squared ensemble and the RTE were shown to agree to leading order. In 12 also the two-point function was derived for the trace squared ensembles and shown to be non-universal using saddle point equations. Here, we will present a scheme to calculate general $k$-point functions of the RTEs in different large- $n$ regimes.

Namely, one has to distinguish two different types of large- $n$ limits the macroscopic and microscopic limit - which may not all be affected by the deformation of the distribution function $\mathcal{P}(M)$. In the example of generalized RTEs which we will study here we find that this is precisely the case. While the macroscopic universality is destroyed by the global constraint, the microscopic correlations at short distances remain unchanged. In a sense, the canonical ensemble $\mathcal{P}(M)$ is replaced by its micro-canonical counterpart $\mathcal{P}_{\delta}(M)$. Therefore we have an explicit model of statistical mechanics at hand, where the correlation functions of both ensembles can be calculated analytically and then be compared for discrepancies. Another remarkable property of RTEs is that they possess a finite support already at finite $n$. Similar to the canonical Gaussian matrix model the RTEs allow for an explicit calculation at finite $n$. This has been shown already in [18] for the one-and two-point function and will be given here for general $k$-point functions. Comparing the finite- $n$ and $n \rightarrow \infty$ results the differences can be thought of as finite-size corrections, when interpreting $n \rightarrow \infty$ as the continuum limit. In the RTE these corrections appear in a different way than in the canonical model, due to the finite support at finite- $n$. Let us explain now in more detail how the large- $n$ limit can be taken.

In the macroscopic large- $n$ limit no restrictions are made on the distance between different eigenvalues. This leads to smooth, universal two- and higher $k$-point correlation functions for the canonical, unconstrained models [3, 迎. Applications can be found in two-dimensional Quantum Gravity [19] as well as the theory of transport properties of mesoscopic wires [2]. For the 
generalized RTEs we have shown in a previous publication together with our collaborators [9], that for a certain class of potentials $V(M)$ the 2-point correlator is no longer universal. In this work we will extend these results to arbitrary polynomial potentials (see also [20]) and to all higher $k$-point resolvents. Since in ref. 9] it was also shown that to leading order all $k$-point resolvents of the $\delta$ - and $\theta$-measure are equivalent we will restrict ourselves here to the former one.

In contrast to that, in the microscopic large- $n$ limit correlations of eigenvalues $\lambda, \mu$ at a distance of the mean level spacing $|\lambda-\mu| \sim 1 / n$ are calculated. It is this kind of limit that finds a wide range of applications in nuclear physics, condensed matter physics (see e.g. [1]) and has initiated exact analytical solutions in the study of Dirac spectra in QCD 21]. We will show that in generalized RTE with purely monomial potential $V(M)=M^{2 p}$ (which includes of course the quadratic case for $p=1$ ) the constraint does not change the local properties at short distances, in the sense that the connected two-point correlator behaves according to the well-known "sine-law" of the Gaussian canonical ensemble [5]. Indeed, this result holds also for higher $k$-point correlation functions. This does not come as a surprise since a global constraint should not change the local properties. We believe that the same is true for more general potentials and that universality holds here as well.

Consequently the present paper is split into two different parts. Section 2 is devoted to the macroscopic large- $n$ limit where we use loop equation techniques, closely following [22, 23]. Although the loop equations are originally designed to calculate higher orders in the $1 / n$-expansion we will restrict ourselves to the planar limit. Since we find non-universality to leading order in all connected correlation functions we do not calculate the likewise nonuniversal higher orders in $1 / n$. The difficulty to deal with the constraint will be treated in a similar way as the situation where the spectral density has a support consisting of several intervals [23]. In these multi-band phases additional constraints have to be imposed to make the solution unique [24, 25]. We will restrict ourselves to hermitian matrices $M$ only, for non-hermitian matrices see [20]. Since only the planar solution is needed for our results they can be easily extended to orthogonal and symplectic matrices using [26]. Extensions to the complex matrix model are straightforward as well since the same loop equation techniques exist in the literature [27, 28]. Very recently finite- $n$ results have been obtained for the eigenvalue density of Gaussian ensembles with real symmetric and complex matrices [29]. In the second part section 3 the microscopic large- $n$ limit is investigated for the 
RTE with a monomial potential $V(M)=M^{2 p}$. Here, we generalize existing results for finite- $n$ of previous publication [18], by improving a technique already used in ref. [9]. Rescaling variables in the microscopic limit and using the inverse Laplace transform we are able to match the connected $k$-point correlator to the well known "sine-law" behavior of the canonical ensemble, thereby proving the microscopic RTE universality. Let us stress that for the RTEs no orthogonal polynomial techniques are applicable.

\section{The macroscopic limit: non-universality}

In order to calculate all correlation functions for the constrained matrix model with an arbitrary potential $V(M)=\sum_{j=1}^{\infty} \frac{g_{j}}{j} M^{j}$ we introduce an auxiliary potential $W(M)$ inside the partition function円

$$
\begin{aligned}
\mathcal{Z}_{\delta} \equiv & \int \mathcal{D} M \exp [-n \operatorname{Tr} W(M)] \delta\left(n^{2} A^{2}-n \operatorname{Tr} V(M)\right) \\
& W(M) \equiv \sum_{j=1}^{\infty} \frac{t_{j}}{j} M^{j}
\end{aligned}
$$

where the two sets of variables $\left\{t_{j}\right\}$ and $\left(\left\{g_{j}\right\}, A\right)$ are taken to be independent. All $k$-point resolvent operators can then be obtained by taking functional derivatives of $\mathcal{Z}_{\delta}$ with respect to $W(p)$ as given below, where we then eventually set the auxiliary potential $W$ to zero at the end. A similar trick has been used in ref. [12] in order to investigate a multi-trace random matrix ensemble [13, 14], showing their non-universality as well. Furthermore we use the complex representation of the $\delta$-function to obtain

$$
\mathcal{Z}_{\delta}=\int \frac{d \alpha}{2 \pi} \int \mathcal{D} M \exp \left[-n \operatorname{Tr}\left(W(M)+i \alpha\left(V(M)-A^{2}\right)\right)\right] .
$$

If we had used instead the following representation of the $\delta$-function, $\delta(x)=$ $\lim _{l \rightarrow \infty} \sqrt{\pi / l} \exp \left[-l x^{2}\right]$, we would have obtained the trace squared ensemble: $\mathcal{P}_{l}(M) \sim \exp \left[2 \ln A^{2} \operatorname{Tr} V(M)-l \operatorname{Tr} V(M)^{2}\right]$, with the strength of the touching interaction being proportional to $l$. However, it is not straightforward to derive and solve loop equations for such an ensemble. In particular, we cannot directly employ the non-universality results of [12], where it was crucial that the single- and multi-trace potentials were different. It is in the form eq. (2.3) that we can actually derive and solve the loop equations for

\footnotetext{
${ }^{1}$ We added a trivial factor of $n^{2}$ inside the delta function.
} 
the constrained model. Throughout the paper the same notation as in [22] is used, which is redisplayed here for completeness. The resolvent or 1-loop correlator is defined as

$$
G(p) \equiv \frac{1}{n}\left\langle\operatorname{Tr} \frac{1}{p-M}\right\rangle_{\delta}=\frac{1}{n} \sum_{k=0}^{\infty} \frac{\left\langle\operatorname{Tr} M^{k}\right\rangle_{\delta}}{p^{k+1}}
$$

and higher $k$-point resolvents are given by

$$
G\left(p_{1}, \ldots, p_{k}\right) \equiv n^{k-2}\left\langle\operatorname{Tr} \frac{1}{p_{1}-M} \cdots \operatorname{Tr} \frac{1}{p_{k}-M}\right\rangle_{\delta, \text { conn }}
$$

where conn stands for the connected part of the expectation value with respect to eq. (2.3). They are defined such that the leading part is of the order $\mathrm{O}(1)$. If we define the free energy $\mathcal{F}_{\delta}$ as follows

$$
\mathcal{Z}_{\delta} \equiv \exp \left[n^{2} \mathcal{F}_{\delta}\right]
$$

all resolvents can be obtained from it by successive applications of the loop insertion operator

$$
\begin{aligned}
\frac{d}{d W}(p) & \equiv-\sum_{j=1}^{\infty} \frac{j}{p^{j+1}} \frac{d}{d t_{j}} \\
G\left(p_{1}, \ldots, p_{k}\right) & =\frac{d}{d W}\left(p_{k}\right) \frac{d}{d W}\left(p_{k-1}\right) \cdots \frac{d}{d W}\left(p_{1}\right) \mathcal{F}_{\delta}+\delta_{k, 1} \frac{1}{p_{1}} .
\end{aligned}
$$

In particular all higher resolvents can be derived from the 1-point resolvent alone.

\subsection{The loop equation in the planar limit}

The loop equation is derived in the usual way from the partition function eq. (2.3) by shifting variables $M \rightarrow M+\epsilon /(p-M)$ and requiring it to be invariant under this shift, i.e. $\left.\frac{d \mathcal{Z}_{\delta}}{d \epsilon}\right|_{\epsilon=0}=0$,

$$
\oint_{\mathcal{C}} \frac{d \omega}{2 \pi i} \frac{V_{e f f}^{\prime}(\omega)}{p-\omega} G(\omega)=G(p)^{2}+\frac{1}{n^{2}} \frac{d}{d W}(p) G(p)
$$

where we have defined the effective potential

$$
V_{e f f}(M)=W(M)+i \bar{\alpha} V(M) .
$$


In eq.(2.9) the integration contour $\mathcal{C}$ encircles the support $[y, x]$ of the spectral density $\rho(\lambda)$ counterclockwise in the complex plane, not including the argument $p \notin[y, x]$. The parameter $i \bar{\alpha}$ inside the effective potential $V_{\text {eff }}$ is determined by the constraint $\langle\operatorname{Tr} V(M)\rangle=n A^{2}$ as a function of all coupling constants, as we will see in more detail below. This constraint can also obtained by requiring the invariance of $\mathcal{Z}_{\delta}$ under the shift $\alpha \rightarrow \alpha+\epsilon$.

Let us stress again that the resolvents are given by differentiating with respect to $W(p)$ and not the effective potential $V_{e f f}(p)$. Due to the $\alpha$-integral in the partition function $\mathcal{Z}_{\delta}$ eq. (2.3) we have $\left\langle\operatorname{Tr} M^{k}\right\rangle_{\delta} \neq\left\langle i \alpha \operatorname{Tr} M^{k}\right\rangle_{\delta}$ which is needed to determine $G(p)$ eq. (2.4). Furthermore let us note that eq. (2.9) looks almost identical to the loop equation of the unconstrained hermitian matrix model [22] defined in the next section eq. (3.1). However, the role of the above mentioned auxiliary potential $W$ as well as the constraint will modify the results of [22]. The constraint leads to similar complications as in the situation where the support of the spectral density consists of several intervals [23].

In order to solve the loop equation we introduce a $1 / n^{2}$ expansion for all $k$-point resolvent operators (2.5)

$$
G\left(p_{1}, \ldots, p_{k}\right)=\sum_{g=0}^{\infty} \frac{1}{n^{2 g}} G_{g}\left(p_{1}, \ldots, p_{k}\right),
$$

where the leading part with genus $g=0$ (planar) is of the order $\mathrm{O}(1)$. In ref. [9] it was shown that for monomial potentials the expectation values of the RTEs possess such an expansion in $1 / n^{2}$ as well. Here we assume that the same holds true for all polynomials potentials. Inserting this expansion into the loop equation (2.9) and taking the large- $n$ limit we obtain to leading order

$$
\oint_{\mathcal{C}} \frac{d \omega}{2 \pi i} \frac{V_{e f f}^{\prime}(\omega)}{p-\omega} G_{0}(\omega)=G_{0}(p)^{2} .
$$

If we make the Ansatz that $G_{0}(p)$ has just one cut in the complex plane or equivalently the support of the eigenvalues consists of the single interval $[y, x]$ we obtain

$$
G_{0}(p)=\frac{1}{2}\left(V_{e f f}^{\prime}(p)-\mathcal{M}(p) \sqrt{(p-x)(p-y)}\right),
$$

where the analytic function $\mathcal{M}(p)$ is given by

$$
\mathcal{M}(p)=\oint_{\mathcal{C}_{\infty}} \frac{d \omega}{2 \pi i} \frac{V_{e f f}^{\prime}(\omega)}{(\omega-p) \sqrt{(\omega-x)(\omega-y)}} .
$$


For details of the derivation see for example ref. [23]. The final result can be written as follows, after deforming back the integration contour,

$$
G_{0}(p)=\frac{1}{2} \oint_{\mathcal{C}} \frac{d \omega}{2 \pi i} \frac{V_{e f f}^{\prime}(\omega)}{p-\omega} \sqrt{\frac{(p-x)(p-y)}{(\omega-x)(\omega-y)}} .
$$

To make the solution complete we still have to determine the endpoints $x$ and $y$ as well as the parameter $i \bar{\alpha}$ in terms of the coupling constants of $W$ and $V$ and the parameter $A$. The first two equations can be obtained from the asymptotic behavior of $G_{0}(p)$. According to the definition (2.4) we have

$$
\lim _{p \rightarrow \infty} G(p)=\frac{1}{p}
$$

Since the leading term does not depend on $n$ it comes from the planar part $G_{0}(p)$ and we obtain the conditions

$$
\begin{aligned}
0 & =\frac{1}{2} \oint_{\mathcal{C}} \frac{d \omega}{2 \pi i} \frac{V_{e f f}^{\prime}(\omega)}{\sqrt{(\omega-x)(\omega-y)}}, \\
1 & =\frac{1}{2} \oint_{\mathcal{C}} \frac{d \omega}{2 \pi i} \frac{\omega V_{e f f}^{\prime}(\omega)}{\sqrt{(\omega-x)(\omega-y)}} .
\end{aligned}
$$

The third equation needed we obtain from the constraint on $\operatorname{Tr} V(M)$ which we rewrite in terms of the spectral density

$$
\begin{aligned}
\rho(\lambda) & =\frac{1}{2 \pi i} \lim _{\epsilon \rightarrow 0}\left(G_{0}(\lambda-i \epsilon)-G_{0}(\lambda+i \epsilon)\right) \\
& =\frac{1}{2 \pi} \mathcal{M}(\lambda) \sqrt{(x-\lambda)(\lambda-y)} .
\end{aligned}
$$

The constraint then reads

$$
A^{2}=\int_{y}^{x} d \lambda \rho(\lambda) V(\lambda)=\int_{y}^{x} \frac{d \lambda}{2 \pi} \mathcal{M}(\lambda) \sqrt{(x-\lambda)(\lambda-y)} V(\lambda),
$$

which determines the parameter $i \bar{\alpha}$ contained in $\mathcal{M}(\lambda)$. This equation together with the boundary conditions eq. (2.17) determines the planar resolvent $G_{0}(p)$ eq. (2.4) completely as a function of the coupling constants of $W$ and $V$ and of the parameter $A$. 


\section{$2.2 \quad$ Higher planar $k$-point resolvents}

Starting from the the planar resolvent $G_{0}(p)$ we can obtain all higher planar $k$-point resolvents by successively applying the loop insertion operator $\frac{d}{d W}$ to it, as it is given in eq. (2.8). Here we use the fact that all resolvents have the same expansion in $1 / n^{2}$ eq. (2.11). For this purpose we introduce a set of new parameters $M_{k}$ and $J_{k}, k \in \mathrm{N}_{+}$. These moments usually play the role of universal parameters of the higher $k$-point resolvents encoding all the information of the potential in addition to the endpoints $x$ and $y$ [22]. We will then rewrite the loop insertion operator in terms of these new variables. This is done in order to make the successive application of $\frac{d}{d W}$ to an algebraic procedure. Finally we calculate explicitly the non-universal planar 2-point resolvent $G_{0}(p, q)$ and comment on the general situation.

Let us begin by defining

$$
\begin{aligned}
M_{k} & \equiv \oint_{\mathcal{C}} \frac{d \omega}{2 \pi i} V_{e f f}^{\prime}(\omega) \frac{\phi(\omega)}{(\omega-x)^{k}}, \quad \phi(\omega) \equiv \frac{1}{\sqrt{(\omega-x)(\omega-y)}}, \\
J_{k} & \equiv \oint_{\mathcal{C}} \frac{d \omega}{2 \pi i} V_{e f f}^{\prime}(\omega) \frac{\phi(\omega)}{(\omega-y)^{k}}, \quad k=1,2, \ldots .
\end{aligned}
$$

Expanding the poles at $x$ and $y$ the moments can be explicitly written as

functions of the coupling constants. Because of $M_{k}=\left.\frac{1}{(k-1) !} \frac{d^{k-1}}{d \lambda^{k-1}} M(\lambda)\right|_{\lambda=x}$ and similarly for $y$ the moments also characterize the multi-critical points of the model. We now rewrite the loop insertion operator eq. (2.7) in the following way

$$
\begin{aligned}
\frac{d}{d W}(p)= & \frac{\partial}{\partial W}(p)+\frac{d x}{d W}(p) \frac{\partial}{\partial x}+\frac{d y}{d W}(p) \frac{\partial}{\partial y}+\frac{d i \bar{\alpha}}{d W}(p) \frac{\partial}{\partial i \bar{\alpha}} \\
& +\sum_{k=1}^{\infty}\left(\frac{d M_{k}}{d W}(p) \frac{\partial}{\partial M_{k}}+\frac{d J_{k}}{d W}(p) \frac{\partial}{\partial J_{k}}\right) \\
\frac{\partial}{\partial W}(p) \equiv & -\sum_{j=1}^{\infty} \frac{j}{p^{j+1}} \frac{\partial}{\partial t_{j}} .
\end{aligned}
$$

While the $\frac{d M_{k}}{d V}(p)$ and $\frac{d J_{k}}{d V}(p)$ can be obtained in a straightforward way from the definition eq. (2.20) (see e.g. [22]) the remaining unknown quantities are derived by applying $\frac{d}{d W}(p)$ to the boundary conditions eqs. (2.17) and (2.19) and solving a linear set of equations. This is done in the Appendix A with the result reading 


$$
\begin{aligned}
M_{1} \frac{d x}{d W}(p) & =\frac{\phi(p)}{p-x}+\frac{4}{B(x-y)}\left(G_{0}(p)-\phi(p)\right) \\
J_{1} \frac{d y}{d W}(p) & =\frac{\phi(p)}{p-y}-\frac{4}{B(x-y)}\left(G_{0}(p)-\phi(p)\right) \\
\frac{1}{i \bar{\alpha}} \frac{d i \bar{\alpha}}{d W}(p) & =-\frac{1}{B}\left(G_{0}(p)-\phi(p)\right)
\end{aligned}
$$

where

$$
\begin{aligned}
B \equiv & i \bar{\alpha} \int_{y}^{x} d \lambda V(\lambda) \frac{1}{2 \pi i} \lim _{\epsilon \rightarrow 0} \times \\
& \times\left[G_{0}(\lambda-i \epsilon)-\phi(\lambda-i \epsilon)-\left(G_{0}(\lambda+i \epsilon)-\phi(\lambda+i \epsilon)\right)\right] .
\end{aligned}
$$

All quantities are expressed by elementary functions and the planar resolvent $G_{0}(p)$ eq. (2.15) and we have set already the auxiliary potential $W \equiv 0$. The result for general $W$ can be derived from eqs. (A.2) and (A.3).

We are now ready to apply the loop insertion operator $\frac{d}{d W}$ in the form (2.21) to $G_{0}(p)$ eq. (2.15), which does not explicitly depend on the moments:

$$
\begin{aligned}
G_{0}(p, q)= & \frac{d}{d W}(q) G_{0}(p) \\
= & \frac{1}{2(p-q)^{2}}\left(\frac{\phi(q)}{\phi(p)}-1\right)+\frac{1}{4(q-p)} \frac{\phi(q)}{\phi(p)}\left(\frac{1}{q-x}+\frac{1}{q-y}\right) \\
& +\frac{d i \bar{\alpha}}{d W}(q) \frac{1}{2} \oint_{\mathcal{C}} \frac{d \omega}{2 \pi i} \frac{V^{\prime}(\omega)}{p-\omega} \sqrt{\frac{(p-x)(p-y)}{(\omega-x)(\omega-y)}} \\
& +\frac{1}{4 \phi(p)}\left(\frac{1}{p-x} M_{1} \frac{d x}{d W}(q)+\frac{1}{p-y} J_{1} \frac{d y}{d W}(q)\right)
\end{aligned}
$$

after performing some contour integrals. If we set $W \equiv 0$ we can use the results eq. (2.23) and finally obtain

$$
G_{0}(p, q)=G_{0}^{c a n}(p, q)-\frac{1}{B}\left(G_{0}(p)-\phi(p)\right)\left(G_{0}(q)-\phi(q)\right),
$$

the planar connected 2-point resolvent of the constrained matrix model. The first part is the well known universal 2-point resolvent of the corresponding unconstrained or canonical matrix model (can) [3]

$$
G_{0}^{c a n}(p, q)=\frac{1}{4(p-q)^{2}}\left(\frac{(p-x)(q-y)+(p-y)(q-x)}{\sqrt{(p-x)(p-y)(q-x)(q-y)}}-2\right)
$$


whereas the second part contains the non-universal terms $G_{0}(p)$ and $G_{0}(q)$. Still, the result is given in closed form for an arbitrary polynomial potential $V(M)$. Eq. (2.26) can be compared with the earlier result [9]

$$
G_{0}(p, q)=G_{0}^{c a n}(p, q)-\frac{1}{2 p} \partial_{p}\left(p G_{0}(p)\right) \partial_{q}\left(q G_{0}(q)\right)
$$

for the special case of monomial potentials $V(M)=M^{2 p}, p \in \mathrm{N}_{+}$. For $p=$ 1,2 we have checked explicitly that the two results eq. (2.26) and eq. (2.28) agree. The corresponding resolvents can be found in 9$]^{2}$ and the parameter $i \bar{\alpha}$ was already determined in [18]. From the procedure described above it is clear that also the higher $k$-point resolvents will remain non-universal since the derivative $\frac{d}{d W}\left(p_{i}\right) G_{0}\left(p_{j}\right)$ always contains terms proportional to $G_{0}\left(p_{i}\right)$ and $G_{0}\left(p_{j}\right)$.

Let us also briefly comment on higher genus contributions. Expanding the loop equation in $1 / n^{2}$ together with eq. 2.11) one obtains for genus one

$$
\left(\hat{\mathcal{K}}-2 G_{0}(p)\right) G_{1}(p)=G_{0}(p, p),
$$

where

$$
\hat{\mathcal{K}} f(p) \equiv \oint_{\mathcal{C}} \frac{d \omega}{2 \pi i} \frac{V_{e f f}^{\prime}(\omega)}{p-\omega} f(\omega) .
$$

The right hand side of eq. 2.29) is easily obtained from eq. 2.26 by setting $p=q$. However, it is no longer a rational function in contrast to $G_{0}^{c a n}(p, p)$. Its non-universality will then translate to $G_{1}(p)$ after inverting the integral operator $\left(\hat{\mathcal{K}}-2 G_{0}(p)\right)$ and thus to higher genera through

$$
\left(\hat{\mathcal{K}}-2 G_{0}(p)\right) G_{g}(p)=\sum_{g \prime=1}^{g-1} G_{g^{\prime}}(p) G_{g-g^{\prime}}(p)+\frac{d}{d W}(p) G_{g-1}(p), \quad g \geq 1 .
$$

For this reason we do not go through the tedious procedure of finding a basis for $\left(\hat{\mathcal{K}}-2 G_{0}(p)\right)$ now including also square roots and inverting it.

\footnotetext{
${ }^{2}$ In eq. (2.18) of ref. 9] the factor of $1 / 2 p$ is missing.
} 


\section{The microscopic limit: universality}

In this section we investigate correlations of eigenvalues at the distance of the mean level spacing $D \sim 1 / n$, the so-called microscopic large- $n$ limit. We will heavily exploit the knowledge about correlations in the unconstrained canonical model for finite as well as infinite $n$. Our main result is an explicit relation between the canonical and RTE correlations for finite- $n$ which then serves to determine the microscopic RTE correlations and prove their universality.

Let us recall the known results about the canonical ensemble which also fixes our notation. The partition function reads

$$
\mathcal{Z} \equiv \int \mathcal{D} M \exp [-n \operatorname{Tr} \tilde{V}(M)]
$$

where $\tilde{V}(M)$ is a polynomial. For the generalized RTE or micro-canonical ensemble

$$
\mathcal{Z}_{\delta} \equiv \int \mathcal{D} M \delta\left(A^{2}-\frac{1}{n} \operatorname{Tr} V(M)\right)
$$

where we do not need to introduce an auxiliary potential in contrast to the previous section. In order to obtain the same microscopic correlations for the two models we will eventually have to relate the coupling constants of the respective polynomial potentials $V$ and $\tilde{V}$ (as in the macroscopic limit, see [18]). The $k$-point density correlation function is defined as

$$
\rho\left(\lambda_{1}, \ldots, \lambda_{k}\right) \equiv\left\langle\frac{1}{n} \operatorname{Tr} \delta\left(\lambda_{1}-M\right) \cdots \frac{1}{n} \operatorname{Tr} \delta\left(\lambda_{k}-M\right)\right\rangle,
$$

and similarly for the delta-measure. Its connected part conn is related in the following way to the $k$-point resolvent defined in the previous section eq. (2.5)

$$
\begin{aligned}
\rho^{c}\left(\lambda_{1}, \ldots, \lambda_{k}\right) \equiv & \left\langle\frac{1}{n} \operatorname{Tr} \delta\left(\lambda_{1}-M\right) \cdots \frac{1}{n} \operatorname{Tr} \delta\left(\lambda_{k}-M\right)\right\rangle_{\text {conn }} \\
= & \frac{1}{n^{2 k-2}}\left(\frac{-1}{2 \pi i}\right)^{k} \times \\
& \times \lim _{\epsilon \rightarrow 0} \sum_{\sigma_{i}= \pm}\left(\prod_{i} \sigma_{i}\right) G\left(\lambda_{1}+\sigma_{1} i \epsilon, \ldots, \lambda_{k}+\sigma_{k} i \epsilon\right)
\end{aligned}
$$

where we have not yet taken the large- $n$ limit. In this section we deal with density correlations instead of resolvents because they can be given more 
explicitly for finite- $n$. Namely in the canonical model all density correlators can be expressed in terms of the Kernel $K_{n}(\lambda, \mu)$ of a set of orthonormal polynomials $P_{l}(\lambda)$ at finite- $n$ [30]

$$
\begin{aligned}
\rho\left(\lambda_{1}, \ldots, \lambda_{k}\right) & =\operatorname{det}_{1 \leq i, j \leq k}\left[K_{n}\left(\lambda_{i}, \lambda_{j}\right)\right], \\
\rho^{c}\left(\lambda_{1}, \ldots, \lambda_{k}\right) & =(-1)^{k+1} \sum_{P} K_{n}\left(\lambda_{1}, \lambda_{2}\right) K_{n}\left(\lambda_{2}, \lambda_{3}\right) \cdots K_{n}\left(\lambda_{k}, \lambda_{1}\right),
\end{aligned}
$$

where the sum is taken over the $(k-1)$ ! distinct cyclic permutation $P$ of the indices $(1,2, \ldots, k)$. The kernel and the polynomials are defined as

$$
\begin{aligned}
K_{n}(\lambda, \mu) & =\frac{1}{n} e^{-\frac{n}{2}(\tilde{V}(\lambda)+\tilde{V}(\mu))} \sum_{k=0}^{n-1} P_{k}(\lambda) P_{k}(\mu), \\
\delta_{k l} & =\int d \lambda e^{-n \tilde{V}(\lambda)} P_{k}(\lambda) P_{l}(\lambda) .
\end{aligned}
$$

The use of the orthogonal polynomial method is only possible for the canonical model because the measure $\exp [-n \operatorname{Tr} \tilde{V}(M)]$ inside the partition function eq. (3.1) factorizes in terms of the eigenvalues $\lambda_{i}$ of the hermitian matrix $M$. For the RTE no such property holds which forces us to seek for other methods. Here we will make use of homogeneity properties for monomial potentials.

Let us finally give the universal results for the canonical ensemble in the microscopic large- $n$ limit, where we restrict ourselves to the origin scaling limit due to the local translational invariance of the canonical ensemble. As mentioned in the beginning we measure eigenvalues in units of the mean level spacing which is $D=1 /(n \rho(0))$ at the origin. Here $\rho(0)$ is the mean eigenvalue density at zero, taken in the macroscopic large- $n$ limit as given in eq. (2.18). We then define new variables $z_{i}=\lambda_{i} / D$ which are kept fixed in the large- $n$ limit. Since $D \rightarrow 0$ as $n \rightarrow \infty$ the variables $\lambda_{i}$ have to go to zero as well. In this particular limit the microscopic correlators are defined as ${ }^{3}$

$$
\rho_{S}\left(z_{1}, \ldots, z_{k}\right) \equiv \lim _{n \rightarrow \infty}(D n)^{k} \rho\left(z_{1} D, \ldots, z_{k} D\right) .
$$

This limit, which is well behaved and finite, can be investigated by using the Darboux-Christoffel formula for the kernel eq. (3.6) and the asymptotic

\footnotetext{
${ }^{3}$ The factor $n^{k}$ appears as we had already defined the $k$-point correlator in eq. (3.3) to be normalized to unity. The appropriate unfolding procedure is usually defined for un-normalized correlators (see e.g. [1]]), which provides us with the correct pre-factor $1 / \rho(0)^{k}$.
} 
large- $n$ behavior of the polynomials $P_{k}(\lambda)$ to obtain [5]

$$
\lim _{n \rightarrow \infty} D_{n} K_{n}\left(z_{1} D, z_{2} D\right)=\frac{\sin \left(\pi\left(z_{1}-z_{2}\right)\right)}{\pi\left(z_{1}-z_{2}\right)} .
$$

This is the universal sine-law which is valid for all polynomial potentials $\tilde{V}(\lambda)$. Together with eqs. (3.5) and (3.7) it completely determines all $k$ point density correlators, connected and not-connected, where at coinciding arguments we have $\lim _{n \rightarrow \infty} D n K_{n}(z D, z D)=1$,

$$
\rho_{S}\left(z_{1}, \ldots, z_{k}\right)=\operatorname{det}_{1 \leq i, j \leq k}\left[\frac{\sin \left(\pi\left(z_{i}-z_{j}\right)\right)}{\pi\left(z_{i}-z_{j}\right)}\right]
$$

and similarly for $\rho_{S}^{c}\left(z_{1}, \ldots, z_{k}\right)$.

We conclude with the following remark. If we had taken the macroscopic large- $n$ limit instead, the connected correlators $\rho^{c}\left(z_{1}, \ldots, z_{k}\right)$ had been of the order $\mathrm{O}\left(1 / n^{2 k-2}\right)$ as one can see from eq. (3.4) together with the fact that the (connected) resolvents are of order $\mathrm{O}(1)$. However, when taking the microscopic limit keeping the $z_{i}$ fixed, the asymptotic kernel eq. (3.8) is of order $\mathrm{O}(1)$ and hence are the connected and not-connected microscopic $k$-point correlators from eq. (3.5). Consequently, the knowledge of both connected and not-connected correlators, is equivalent here since they can be obtained from each other by adding or subtracting $l$-point correlators $(l<k)$ of order $\mathrm{O}(1)$. In that sense the microscopic large- $n$ limit modifies the usual large- $n$ factorization of correlation functions.

\subsection{Microscopic $k$-point correlation functions}

As it has been mentioned already the correlation functions in the RTE can not be calculated using orthogonal polynomials because of the Dirac $\delta$ function in the measure. However, in the particular case of purely monomial potentials $V(M)=M^{2 p}$, the evaluation of the connected $k$-point correlator in the microscopic limit is straightforward. We shall exploit some homogeneity properties of this case, which make it possible to relate ensemble averages in the monomial RTE to ensemble averages of the same quantities in the canonical ensemblef with potential $\tilde{V}(M)=g M^{2 p}$. Let us define a function $F_{k}(M ; \lambda)$ of the matrix $M$ and of a set of parameters $\lambda=\left(\lambda_{1}, \ldots, \lambda_{k}\right)$, that

\footnotetext{
${ }^{4}$ This technique is a slight generalization of ref. 9], where only the macroscopic limit was investigated.
} 
satisfies the following homogeneity property under a rescaling of the matrix

$$
F_{k}(t M ; \lambda)=t^{a} F_{k}\left(M ; t^{b} \lambda\right) \quad \text { for } \quad t, a, b \in \mathrm{R} .
$$

A simple example for such a function is the operator $\operatorname{Tr} \delta(\lambda-M) / n$ which has $a=b=-1$. Also any such product is a homogeneous function, as inside the average of the $k$-point correlation function eq. (3.3), having $a=-k$ and $b=-1$. In appendix $B$ we derive the following formula for such homogeneous functions $F_{k}$, which relates their canonical and RTE average

$$
\left\langle F_{k}(M ; \lambda)\right\rangle_{\delta}=\frac{\left(g n^{2}\right)^{\frac{a}{2 p}} \Gamma\left(\frac{n^{2}}{2 p}\right)}{A^{\frac{n^{2}}{p}-2}} \mathcal{L}^{-1}\left[\frac{\left\langle F_{k}\left(M ;\left(\frac{g n^{2}}{t}\right)^{\frac{b}{2 p}} \lambda\right)\right\rangle}{t^{\frac{n^{2}+a}{2 p}}}\right]\left(A^{2}\right) .
$$

Here $\mathcal{L}^{-1}[h(t)](x)$ is the inverse Laplace transform of a function $h(t)$, evaluated at the point $x>0$ (for an integral representation see eq. (B.5)). Eq. (3.11) holds for any finite $n$. If we choose the 1- or 2-point correlator from eq. (3.3) as an example we reproduce the finite- $n$ results of ref. [18] for $\rho_{\delta}(\lambda)$ and $\rho_{\delta}(\lambda, \mu)$ which were given in the case of a Gaussian potential $p=1$. If we choose for general $k$ to take $\left\langle F_{k}(M ; \lambda)\right\rangle_{\delta}=\rho_{\delta}\left(\lambda_{1}, \ldots, \lambda_{k}\right)$, which obviously fulfills the criterion (3.10), we obtain from eq. (3.11) the following expression for the $k$-point RTE correlator

$\rho_{\delta}\left(\lambda_{1}, \ldots, \lambda_{k}\right)=\frac{\left(g n^{2}\right)^{\frac{-k}{2 p}} \Gamma\left(\frac{n^{2}}{2 p}\right)}{A^{\frac{n^{2}}{p}}-2} \mathcal{L}^{-1}\left[\frac{\rho\left(\left(\frac{g n^{2}}{t}\right)^{\frac{-1}{2 p}} \lambda_{1}, \ldots,\left(\frac{g n^{2}}{t}\right)^{\frac{-1}{2 p}} \lambda_{k}\right)}{t^{\frac{n^{2}-k}{2 p}}}\right]\left(A^{2}\right)$

Now we make use of the fact that at finite $n$ the correlations $\rho\left(\lambda_{1}, \ldots, \lambda_{k}\right)$ from eq. (3.5) can be written as a polynomial in all variables $\lambda_{i}$ times an exponential measure factor:

$$
\rho\left(\lambda_{1}, \ldots, \lambda_{k}\right) \equiv e^{-n g \sum_{i=1}^{k} \lambda_{i}^{2 p}} \sum_{l_{1}, \ldots, l_{k}=0}^{2 n-2} c_{\left\{l_{1}, \ldots, l_{k}\right\}}^{(n)} \lambda_{1}^{l_{1}} \cdots \lambda_{k}^{l_{k}}
$$

Due to this fact we can actually perform the inverse Laplace transformation 


$$
\begin{aligned}
\rho_{\delta}\left(\lambda_{1}, \ldots, \lambda_{k}\right)= & \frac{\Gamma\left(\frac{n^{2}}{2 p}\right)}{\left(g n^{2}\right)^{\frac{k}{2 p}} A^{\frac{n^{2}}{p}-2}} \sum_{l_{1}, \ldots, l_{k}=0}^{2 n-2} \frac{c_{\left\{l_{1}, \ldots, l_{k}\right\}}^{(n)}}{\left(g n^{2}\right)^{\frac{1}{2 p} \Sigma_{i} l_{i}}} \lambda_{1}^{l_{1}} \cdots \lambda_{k}^{l_{k}} \times \\
& \times \mathcal{L}^{-1}\left[t^{-\frac{n^{2}-k-\Sigma_{i} l_{i}}{2 p}}\right]\left(A^{2}-\frac{1}{n} \sum_{i=1}^{k} \lambda_{i}^{2 p}\right) \\
= & \frac{\theta\left(A^{2}-\frac{1}{n} \sum_{i=1}^{k} \lambda_{i}^{2 p}\right)}{\left(g n^{2}\right)^{\frac{k}{2 p}} A^{\frac{n^{2}}{p}}-2} \sum_{l_{1}, \ldots, l_{k}=0}^{2 n-2} \frac{c_{\left\{l_{1}, \ldots, l_{k}\right\}}^{(n)} \lambda_{1}^{l_{1}} \cdots \lambda_{k}^{l_{k}}}{\left(g n^{2}\right)^{\frac{1}{2 p} \Sigma_{i} l_{i}}} \times \\
& \times \frac{\Gamma\left(\frac{n^{2}}{2 p}\right)}{\Gamma\left(\frac{n^{2}-k-\Sigma_{i} l_{i}}{2 p}\right)}\left(A^{2}-\frac{1}{n} \sum_{i=1}^{k} \lambda_{i}^{2 p}\right)^{\frac{n^{2}-k-\Sigma_{i} l_{i}}{2 p}-1},(3.14)
\end{aligned}
$$

where we have used the shift property $\mathcal{L}^{-1}\left[h(t) e^{\sigma t}\right](x)=\mathcal{L}^{-1}[h(t)](x+\sigma)$, the linearity of the inverse Laplace transform and eq. (B.7) of Appendix B. Eq. (3.14) is our first result, the finite- $n k$-point correlation function for RTEs with monomial potential $V(M)=M^{2 p}$ in terms of the corresponding canonical correlator at finite- $n$ with potential $\tilde{V}(M)=g M^{2 p}$. In general, eqs. (3.13) and (3.14) are different from each other at finite- $n$. This remains true in the macroscopic large- $n$ limit, as we have seen in the previous section. In the remaining part we will show that in the microscopic large- $n$ limit, however, they happen to coincide.

In a first step, we determine the mean level spacing $D_{\delta}=1 /\left(n \rho_{\delta}(0)\right)$ in order to define the appropriate microscopic scaling limit. From eq. (3.14) at $\lambda=0$ one can read off $\rho_{\delta}(0)$, since then the sum over all $l_{i}$ collapses. We obtain

$$
\rho_{\delta}(0)=\frac{c_{\{0\}}^{(n)}}{\left(g n^{2} A^{2}\right)^{\frac{1}{2 p}}} \frac{\Gamma\left(\frac{n^{2}}{2 p}\right)}{\Gamma\left(\frac{n^{2}-1}{2 p}\right)} \longrightarrow \frac{c_{\{0\}}}{\left(2 p g A^{2}\right)^{\frac{1}{2 p}}}
$$

for its large- $n$ value, with $c_{\{0\}}=\rho(0)$ being the macroscopic large- $n$ limit of the canonical spectral density eq. (2.18) at the origin (which exists and is finite). In order to have the same mean level spacing as in the canonical ensemble we would have to set $2 p g A^{2}=1$. This identification of coupling constants occurs also in the macroscopic large- $n$ limit in order to match the corresponding macroscopic spectral densities (see refs. [9, 18]). However, since we measure all correlations in units of $D$ and $D_{\delta}$ respectively we do not need to identify $D=D_{\delta}$ since they drop out in the microscopic correlators anyway, as we will see below. 
We will now take the microscopic limit analogue to eq. (3.7) with rescal$\operatorname{ing} \lambda_{i}=z_{i} D_{\delta}$ of our finite- $n$ relation (3.14). The large- $n$ limit of the different factors can be obtained as follows, starting with the $\theta$-function term in eq. (3.14)

$$
\theta\left(A^{2}-\frac{2 p g A^{2}}{n^{2 p+1}} \sum_{i=1}^{k}\left(\frac{z_{i}}{c_{\{0\}}}\right)^{2 p}\right) \longrightarrow \theta\left(A^{2}\right)=1
$$

The remaining $A$-dependent terms yield

$$
\begin{aligned}
& A^{-\frac{n^{2}}{p}+2}\left(A^{2}-\frac{2 p g A^{2}}{n^{2 p+1}} \sum_{i=1}^{k}\left(\frac{z_{i}}{c_{\{0\}}}\right)^{2 p}\right)^{\frac{n^{2}-k-\Sigma_{i} l_{i}}{2 p}-1}= \\
& =A^{-\frac{1}{p}\left(k+\Sigma_{i} l_{i}\right)}\left(1-\left[\frac{n^{2}-k-\Sigma_{i} l_{i}}{2 p}-1\right] \frac{2 p g}{n^{2 p+1}} \sum_{i=1}^{k}\left(\frac{z_{i}}{c_{\{0\}}}\right)^{2 p}+\ldots\right) \\
& =A^{-\frac{1}{p}\left(k+\Sigma_{i} l_{i}\right)}\left(1+\mathrm{O}\left(\frac{1}{n^{2 p-1}}\right)\right)
\end{aligned}
$$

which still has to be evaluated under the sum over $l_{i}$ 's. Here we have used in the second step that the first factor inside the parenthesis is of $\mathrm{O}\left(n^{2}\right)$ since

$$
\sum_{i=1}^{k} l_{i} \leq k(2 n-2)
$$

is at most of $\mathrm{O}(n)$. Because of $p \geq 1$ the corrections are sub-leading. The factor containing $\Gamma$-functions we evaluate together with the explicit factors of $n$ in eq. (3.14)

$$
\begin{aligned}
& \frac{\Gamma\left(\frac{n^{2}}{2 p}\right)}{\left(g n^{2}\right)^{\frac{1}{2 p}\left(k+\Sigma_{i} l_{i}\right)} \Gamma\left(\frac{n^{2}}{2 p}-\frac{\left(k+\Sigma_{i} l_{i}\right)}{2 p}\right)}= \\
& =(2 p g)^{-\frac{1}{2 p}\left(k+\Sigma_{i} l_{i}\right)}\left(1-\frac{1}{4 p n^{2}}\left(k+\sum_{i=1}^{k} l_{i}\right)\left(2 p+k+\sum_{i=1}^{k} l_{i}\right)+\ldots\right)
\end{aligned}
$$

It remains to be shown that the second term and thus higher terms in the expansion are sub-leading. A naive counting from eq. (3.18) suggests that this might not be the case. In order to use the microscopic results for the canonical correlators eq. (3.9) we put together our results obtained so far 


$$
\begin{aligned}
& \lim _{n \rightarrow \infty}\left(D_{\delta} n\right)^{k} \rho_{\delta}\left(z_{1} D_{\delta}, \ldots, z_{k} D_{\delta}\right)= \\
& =\lim _{n \rightarrow \infty} \frac{\left(2 p g A^{2}\right)^{\frac{k}{2 p}}}{\left(c_{\{0\}}\right)^{k}} \sum_{l_{1}, \ldots, l_{k}=0}^{2 n-2} c_{\left\{l_{1}, \ldots, l_{k}\right\}}^{(n)}\left(\frac{z_{1}\left(2 p g A^{2}\right)^{\frac{1}{2 p}}}{n c_{\{0\}}}\right)^{l_{1}} \cdots\left(\frac{z_{k}\left(2 p g A^{2}\right)^{\frac{1}{2 p}}}{n c_{\{0\}}}\right)^{l_{k}} \\
& \times\left(2 p g A^{2}\right)^{-\frac{1}{2 p}\left(k+\Sigma_{i} l_{i}\right)}\left(1-\frac{1}{4 p n^{2}}\left(k+\sum_{i=1}^{k} l_{i}\right)\left(2 p+k+\sum_{i=1}^{k} l_{i}\right)+\ldots\right) \\
& =\lim _{n \rightarrow \infty}\left(1-\frac{1}{4 p n^{2}}\left(\sum_{i=1}^{k} \partial_{z_{i}} z_{i}\right)\left(2 p+\sum_{i=1}^{k} \partial_{z_{i}} z_{i}\right)+\ldots\right) \\
& \times \frac{1}{\left(c_{\{0\}}\right)^{k}} \sum_{l_{1}, \ldots, l_{k}=0}^{2 n-2} c_{\left\{l_{1}, \ldots, l_{k}\right\}}^{(n)}\left(\frac{z_{1}}{n c_{\{0\}}}\right)^{l_{1}} \cdots\left(\frac{z_{k}}{n c_{\{0\}}}\right)^{l_{k}} .
\end{aligned}
$$

Here, the pre-factors from eqs. (3.17) and (3.19) have canceled with the factors of $\left(2 p g A^{2}\right)^{\frac{1}{2 p}}$ from the unfolding. Now we know from the canonical ensemble eqs. (3.13), (3.7) and (3.9) that the limit of the sum over the $l_{i}$ exists and is finite:

$$
\rho_{S}\left(z_{1}, \ldots, z_{k}\right)=\lim _{n \rightarrow \infty} \frac{1}{\left(c_{\{0\}}\right)^{k}} \sum_{l_{1}, \ldots, l_{k}=0}^{2 n-2} c_{\left\{l_{1}, \ldots, l_{k}\right\}}^{(n)}\left(\frac{z_{1}}{n c_{\{0\}}}\right)^{l_{1}} \cdots\left(\frac{z_{k}}{n c_{\{0\}}}\right)^{l_{k}} .
$$

Hence the term in eq. (3.20) proportional to $1 /\left(4 p n^{2}\right)$ is indeed sub-leading and we have as a final result

$$
\rho_{\delta, S}\left(z_{1}, \ldots, z_{k}\right)=\rho_{S}\left(z_{1}, \ldots, z_{k}\right)
$$

or more explicitly in terms of eq. (3.9). Since our derivation holds for the RTE with an arbitrary monomial potential $V(M)=M^{2 p}$, we have not only derived all $k$-point correlation functions but also proved their universality for the given class of potentials. Let us finally point out that the equivalence eq. (3.22) also holds for the corresponding connected $k$-point correlation functions. As we have mentioned already at the end of the previous subsection, they are of the same order in the microscopic limit and they can be obtained from each other by adding or subtracting lower $l$-point correlators. 


\section{Conclusions}

We have shown for RTEs as an example of constrained random matrix models that in one and the same model correlation functions may exhibit universal and non-universal behavior in different large- $n$ regimes. In particular, in the macroscopic large- $n$ limit all planar connected $k$-point resolvents are non-universal and a closed expression was given for the resolvent $G_{0}(z, w)$ for an arbitrary potential. Hence when switching from the canonical to the RTE or micro-canonical ensemble, the delta-function constraint destroys the macroscopic universality.

A different behavior appears in the study of correlations of eigenvalues at the scale of the mean level spacing $1 / n$. Here we recover the sine-law of the canonical ensemble and prove its universality for the class of RTEs with monomial potential. This leads us to conjecture that microscopic universality holds also for more general RTEs. The result in the microscopic limit is not unexpected since a global constraint should not influence the local statistics of eigenvalues.

Acknowledgments: We wish to thank G. Cicuta and L. Molinari for their very enjoyable collaboration and many discussions on work done prior to this publication. Furthermore one of us (G.A.) wishes to thank the Physics Department of Parma for its hospitality extended to him on several occasions. The work of G.V. is supported in part by MURST within the project of "Theoretical Physics of fundamental Interactions".

\section{A The functions $\frac{d x}{d W}(p), \frac{d y}{d W}(p)$ and $\frac{d i \bar{\alpha}}{d W}(p)$}

In this Appendix we apply the operator $\frac{d}{d W}(p)$ eq. (2.21) to the boundary conditions eqs. (2.17) and (2.19) and solve the linear set of equations for the quantities $\frac{d x}{d W}(p), \frac{d y}{d W}(p)$ and $\frac{d i \bar{\alpha}}{d W}(p)$. Using the identity

$$
\frac{\partial}{\partial W}(p) W^{\prime}(\omega)=\frac{-1}{(p-\omega)^{2}}
$$

we obtain

$$
0=\partial_{p} \phi(p)+\frac{1}{2}\left(M_{1} \frac{d x}{d W}(p)+J_{1} \frac{d y}{d W}(p)\right)+\oint_{\mathcal{C}} \frac{d \omega}{2 \pi i} V^{\prime}(\omega) \phi(\omega) \frac{d i \bar{\alpha}}{d W}(p)
$$




$$
\begin{aligned}
0= & \partial_{p}(p \phi(p))+\frac{1}{2}\left(x M_{1} \frac{d x}{d W}(p)+y J_{1} \frac{d y}{d W}(p)\right) \\
& +\oint_{\mathcal{C}} \frac{d \omega}{2 \pi i} \omega V^{\prime}(\omega) \phi(\omega) \frac{d i \bar{\alpha}}{d W}(p)
\end{aligned}
$$

from eq. (2.17) and

$$
\begin{aligned}
0= & \frac{C}{2}\left(M_{1} \frac{d x}{d W}(p)-\frac{\phi(p)}{p-x}\right)+\frac{D}{2}\left(J_{1} \frac{d x}{d W}(p)-\frac{\phi(p)}{p-y}\right) \\
& +\frac{d i \bar{\alpha}}{d W}(p) \int_{y}^{x} \frac{d \lambda}{2 \pi} V(\lambda) \sqrt{(x-\lambda)(\lambda-y)} \oint_{\mathcal{C}} \frac{d \omega}{2 \pi i} \frac{V^{\prime}(\omega)}{\omega-\lambda} \phi(\omega) \\
& +\frac{1}{2} \oint_{\mathcal{C}} \frac{d \omega}{2 \pi i} \frac{V^{\prime}(\omega)}{p-\omega} \sqrt{\frac{(\omega-x)(\omega-y)}{(p-x)(p-y)}},
\end{aligned}
$$

from eq. (2.19) after some calculation. Here we have introduced

$$
\begin{aligned}
C & \equiv \int_{y}^{x} \frac{d \lambda}{2 \pi} \frac{V(\lambda)}{x-\lambda} \sqrt{(x-\lambda)(\lambda-y)} \\
D & \equiv \int_{y}^{x} \frac{d \lambda}{2 \pi} \frac{V(\lambda)}{y-\lambda} \sqrt{(x-\lambda)(\lambda-y)} .
\end{aligned}
$$

We note that special care has to be taken when applying $\frac{d}{d W}(p)$ to $\mathcal{M}(\lambda)$ which is then no longer an analytic function in $\lambda$. Therefore the contour $\mathcal{C}_{\infty}$ has to be deformed in eq. (2.14) to contain only the pole and cut in the integrand, and not the new pole introduced by $\frac{d}{d W}(p)$ (see also Appendix $A$ in [23]). The linear set of equations eq. (A.2) and eq. (A.3) simplifies considerably when setting $W \equiv 0$ because of $V_{\text {eff }}(\omega)=i \bar{\alpha} V(\omega)$ in that case. It is this limit which we will need in order give a closed final expression for the planar 2-point resolvent of the pure delta-measure without the auxiliary potential in eq. (2.1). Using eq. (2.17) it follows

$$
\begin{aligned}
0= & M_{1} \frac{d x}{d W}(p)-\frac{\phi(p)}{p-x}+J_{1} \frac{d x}{d W}(p)-\frac{\phi(p)}{p-y} \\
0= & \frac{x}{2}\left(M_{1} \frac{d x}{d W}(p)-\frac{\phi(p)}{p-x}\right)+\frac{y}{2}\left(J_{1} \frac{d x}{d W}(p)-\frac{\phi(p)}{p-y}\right)+\frac{2}{i \bar{\alpha}} \frac{d i \bar{\alpha}}{d W}(p) \\
0= & \frac{C}{2}\left(M_{1} \frac{d x}{d W}(p)-\frac{\phi(p)}{p-x}\right)+\frac{D}{2}\left(J_{1} \frac{d x}{d W}(p)-\frac{\phi(p)}{p-y}\right) \\
& +\frac{1}{i \bar{\alpha}} A^{2} \frac{d i \bar{\alpha}}{d W}(p)+\frac{1}{i \bar{\alpha}}\left(G_{0}(p)-\phi(p)\right) .
\end{aligned}
$$


This can be easily solved for the desired quantities. We obtain

$$
\begin{aligned}
M_{1} \frac{d x}{d W}(p) & =\frac{\phi(p)}{p-x}+\frac{4}{B(x-y)}\left(G_{0}(p)-\phi(p)\right) \\
J_{1} \frac{d y}{d W}(p) & =\frac{\phi(p)}{p-y}-\frac{4}{B(x-y)}\left(G_{0}(p)-\phi(p)\right) \\
\frac{1}{i \bar{\alpha}} \frac{d i \bar{\alpha}}{d W}(p) & =-\frac{1}{B}\left(G_{0}(p)-\phi(p)\right),
\end{aligned}
$$

as given in eq. (2.23) where the following abbreviation has been introduced

$$
B=i \bar{\alpha}\left(A^{2}+2 \frac{C+D}{x-y}\right) .
$$

One can easily convince oneself that it equals the form given in eq. (2.24).

\section{B RTE via inverse Laplace transform}

In this appendix we derive eq. (3.11) which expresses expectation values with respect to the delta-measure in terms of averages with respect to the canonical measure eq. (3.1) using the inverse Laplace transform. Let $F_{k}(M ; \lambda)$ be a function of the Hermitian $n \times n$ matrix $M$ and of the set of parameters $\lambda=\left(\lambda_{1}, \ldots, \lambda_{k}\right)$, such that it satisfies the homogeneity property

$$
F_{k}(t M ; \lambda)=t^{a} F_{k}\left(M ; t^{b} \lambda\right)
$$

for some real $t, a$ and $b$. In other words $F_{k}$ is a homogeneous function of degree $a$ with respect to matrix elements $M_{i j}$ and of degree $(-b)$ with respect to each parameter $\lambda_{i}$. An example for such a function is the operator inside the average of eq. (3.3). The matrix integral considered in this appendix is:

$$
I\left[F_{k}\right]=\int \mathcal{D} M \delta\left(A^{2}-\frac{1}{n} \operatorname{Tr}\left[M^{2 p}\right]\right) F_{k}(M ; \lambda),
$$

with $p$ an integer number. It is proportional to the average $\left\langle F_{k}\right\rangle_{\delta}$. Introducing the complex representation of the delta function $2 \pi \delta(x)=\int d y \exp [i x y]$ and then scaling all matrix elements by a factor $\left(g n^{2} /\left(i y+0^{+}\right)\right)^{1 / 2 p}$, eq. (B.2) reads:

$I\left[F_{k}\right]=\int_{-\infty}^{+\infty} \frac{d y}{2 \pi} e^{i y A^{2}}\left(\frac{g n^{2}}{i y+0^{+}}\right)^{\frac{n^{2}}{2 p}} \int \mathcal{D} M e^{-n g \operatorname{Tr}\left[M^{2 p}\right]} F_{k}\left(\left(\frac{g n^{2}}{i y+0^{+}}\right)^{\frac{1}{2 p}} M ; \lambda\right)$ 
where we have interchanged integrals. By using the homogeneity property (B.1) we can rewrite the matrix integral as a canonical ensemble average, up to the normalization factor $\mathcal{Z}$ eq. (3.1):

$$
\begin{aligned}
I\left[F_{k}\right] & =\mathcal{Z} \int_{-\infty}^{+\infty} \frac{d y}{2 \pi} e^{i y A^{2}}\left(\frac{g n^{2}}{i y+0^{+}}\right)^{\frac{n^{2}+a}{2 p}}\left\langle F_{k}\left(M ;\left(\frac{g n^{2}}{i y+0^{+}}\right)^{\frac{b}{2 p}} \lambda\right)\right\rangle \\
& =\mathcal{Z}\left(g n^{2}\right)^{\frac{n^{2}+a}{2 p}} \mathcal{L}^{-1}\left[\frac{\left\langle F_{k}\left(M ;\left(\frac{g n^{2}}{t}\right)^{\frac{b}{2 p}} \lambda\right)\right\rangle}{t^{\frac{n^{2}+a}{2 p}}}\right]\left(A^{2}\right) .
\end{aligned}
$$

In the last step we used the complex representation of the inverse Laplace transform, i.e.

$$
\mathcal{L}^{-1}[h(t)](x)=\frac{1}{2 \pi i} \int_{-i \infty+0^{+}}^{+i \infty+0^{+}} d t e^{t x} h(t), x>0 .
$$

In order to obtain the correct normalization of the delta-average we evaluate eq. (B.4) in the case $F=1$ (with $a=b=0$ ):

$$
I[1]=\left(g n^{2}\right)^{\frac{n^{2}}{2 p}} \frac{A^{\frac{n^{2}}{p}-2}}{\Gamma\left(\frac{n^{2}}{2 p}\right)} \mathcal{Z},
$$

where we used the formula

$$
\mathcal{L}^{-1}\left[\frac{1}{t^{\gamma+1}}\right](x)=\frac{x^{\gamma}}{\Gamma(\gamma+1)} \theta(x), \operatorname{Re}(\gamma)>-1 .
$$

Finally, by putting together eqs. (B.4) and (B.6), the ensemble average $\left\langle F_{k}\right\rangle_{\delta} \equiv I\left[F_{k}\right] / I[1]$ with respect to the measure $\delta\left(A^{2}-\operatorname{Tr}\left[M^{2 p}\right] / n\right)$ reads:

$$
\left\langle F_{k}\right\rangle_{\delta}=\frac{\left(g n^{2}\right)^{\frac{a}{2 p}}}{A^{\frac{n^{2}}{p}}-2} \Gamma\left(\frac{n^{2}}{2 p}\right) \mathcal{L}^{-1}\left[\frac{\left\langle F_{k}\left(M ;\left(\frac{g n^{2}}{t}\right)^{\frac{b}{2 p}} \lambda\right)\right\rangle}{t^{\frac{n^{2}+a}{2 p}}}\right]\left(A^{2}\right)
$$

which is just eq. (3.11). 


\section{References}

[1] T. Guhr, A. Müller-Groeling and H.A. Weidenmüller, Phys. Rep. 299 (1998) 190.

[2] C.W. Beenakker, Rev. Mod. Phys. 69 (1997) 731.

[3] J. Ambjørn, J. Jurkiewicz and Y. Makeenko, Phys. Lett. B251 (1990) 517.

[4] G. Akemann and J. Ambjørn, J. Phys. A29 (1996) L555.

[5] E. Brézin and A. Zee, Nucl. Phys. B402 (1993) 613.

[6] G. Akemann, P. H. Damgaard, U. Magnea and S. Nishigaki, Nucl. Phys. B487 (1997) 721.

[7] M.J. Bowick and E. Brézin, Phys. Lett. B268 (1991) 21.

[8] E. Kanzieper and V. Freilikher, Phys. Rev. E55 (1997) 3712.

[9] G. Akemann, G.M. Cicuta, L. Molinari and G. Vernizzi, Phys. Rev. E60 (1999) 5287.

[10] A. Khorunzhy, B. Khoruzhenko and L. Pastur, J. Phys. A28 (1995) L31; J. Math. Phys. 37 (1996) 5033.

[11] J. D'Anna and A. Zee, Phys. Rev. E53 (1996) 1399.

[12] S. Iso and A. Kavalov, Nucl. Phys. B501 (1997) 670.

[13] S.R. Das, A. Dhar, A.M. Sengupta and S.R. Wadia, Mod. Phys. Lett. A5 (1990) 1041.

[14] G.M. Cicuta and E. Montaldi, Mod. Phys. Lett. A5 (1990) 1927.

[15] N. Rosenzweig, Statistical Physics, Brandeis Summer Institute 1962, G. Uhlenbeck et al., W.A. Benjamin Inc. 1963.

[16] B.V. Bronk, Princeton University thesis 1964, as cited in 30, Chapter 19.

[17] G.P. Korchemsky, Mod. Phys. Lett. A7 (1992) 3081. 
[18] G. Akemann, G.M. Cicuta, L. Molinari and G. Vernizzi, Phys. Rev. E59 (1999) 1489.

[19] J. Ambjørn, B. Durhuus and T. Jonsson, Quantum Geometry, Cambridge University Press 1997.

[20] G. Vernizzi, Classes of Universality in Random Matrix Theory, PhDthesis, Parma 1999.

[21] E.V. Shuryak and J.J.M. Verbaarschot, Nucl. Phys. A560 (1993) 306.

[22] J. Ambjørn, L. Chekhov, C. F. Kristjansen and Yu. Makeenko, Nucl. Phys. B404 (1993) 127.

[23] G. Akemann Nucl. Phys. B482 (1996) 403.

[24] F. David, Nucl. Phys. B348 (1991) 507.

[25] J. Jurkiewicz, Phys. Lett. B245 (1990) 178.

[26] C. Itoi, Nucl. Phys. B493 (1997) 651.

[27] J. Ambjørn, C.F. Kristjansen and Yu. Makeenko, Mod. Phys. Lett. A7 (1992) 3187.

[28] G. Akemann, Nucl. Phys. B507 (1997) 475.

[29] R. Delannay and G. Le Caër, J. Phys. A33 (2000) 2611.

[30] M.L. Mehta, Random Matrices, Academic Press 1991. 